\title{
Study of the Effect of Adding Letrozole to Gonadotropin-Releasing Hormone Antagonist Protocol in Poor Responders Undergoing Intracytoplasmic Sperm Injection
}

\author{
Original \\ Article \\ Suzan Samir ElSharkawy ${ }^{I}$, Mohamed Salah Al Din Abd Raboo ${ }^{1}$, Mohamed \\ Murad Al Abd', Safaa Mohamed Khamis Mohamed ${ }^{2}$ \\ ${ }^{1}$ Department of Obstetrics and Gynecology, Faculty of Medicine, Alexandria University \\ ${ }^{2}$ Department of Obstetrics and Gynecology, Dar Ismail Maternity Hospital, Alexandria, Egypt
}

\begin{abstract}
Background: Poor responders constitute a less fortunate group of patients attending any IVF/ICSI clinic. Identifying these patients helps in choosing the most appropriate stimulation protocol.

Aim:This is a randomized controlled prospective study that aimed to study the effect of adding letrozole to GnRH antagonist protocol in poor responders undergoing ICSI.

Materials and Methods: This study was carried out on a sample of 70 infertile couple, thirty five patients in each group, five cases were canceled in group I (letrozole/antagonist group), while six patients in group II (antagonist group) canceled the cycle. In group I, letrozole $5 \mathrm{mg}$ was given from day 2 cycle for 5 days. Initial dose of HMG 300 I.U from day three cycle, then the dose was modulated according to response in both groups and ICSI was done. The women were followed to detect the occurrence of pregnancy by measuring serum B-hCG 14 day after embryo transfer and clinical pregnancy was followed up at 6 th week of gestation.

Results: The dose of HMG was significantly lower in group I than in group II, while there were no significant statistical differences in the duration of stimulation, number of oocytes retrieved, fertilization rate, implantation rate, cycle cancelation rate, and clinical pregnancy rate with adding letrozole to GnRH antagonist cycles in women with POR.

Conclusion: Letrozole is recommended to be added to ICSI stimulation protocols in poor responders. Although it didn't affect the pregnancy rate, letrozole decreases the dose and the cost of ICSI cycles.
\end{abstract}

Received: 23 August 2019, Accepted: 27 September 2019

Key Words: GnRH antagonists, ICSI cycles, letrozole, poor responders

Corresponding Author: Suzan Samir ElSharkawy, Department of Obstetrics and Gynecology, Faculty of Medicine, Alexandria University, Egypt, Tel.: +201090052715, E-mail: s_abdelrahim00@alexmed.edu.eg

ISSN: 2090-7265, August 2021, Vol.11, No. 3

\section{INTRODUCTION}

Over the last three decades, assisted reproductive technologies (ART) have improved dramatically; however handling women with poor ovarian reserve (POR) is considered one of the most difficult tasks for subfertility clinic $^{[1,2]}$.

To diagnose POR, we need at least one stimulation cycle with unsatisfactory results. With adequate gonadotropin stimulation there will be low estradiol (E2) levels, a decreased number of mature follicles, the retrieval of scanty oocytes, and sometimes cancellation of the whole (IVF) cycle ${ }^{[3,4]}$.

In cases of advanced maternal age over 40 years, low antral follicle count (AFC), a high follicle stimulating hormone (FSH) level in early follicular phase, a low antimüllerian hormone (AMH) level and low inhibin B, we can anticipate POR in superovulation IVF cycles ${ }^{[5]}$.
The European Society for Human Reproduction and Embryology (ESHRE) consensus in 2011 represented the Bologna criteria to standardize the definition of POR. To define poor response, at least two of the following three criteria must be present ; advanced maternal age ( $\geq 40$ years) or any other risk factor for POR. Previous POR $(\leq 3)$ oocytes with a conventional stimulation protocol. An abnormal ovarian reserve test (i.e. AFC $<5-7$ follicles or AMH $<0.5-1.1 \mathrm{ng} / \mathrm{ml})^{[6]}$.

A poor ovarian response (defined as $\leq 4$ oocytes retrieved per cycle of controlled ovarian stimulation (COS) is estimated to be almost $20 \%$ of all women undergoing IVF/ ICSI, which increases the drop-out rates, and decreases the live birth rates when compared to women with a normal response $\mathrm{e}^{[7,8]}$.

What is the optimal stimulation protocol for poor responder patients? To answer this challenging question; 
many modalities have been developed such as: high dose gonadotropins administration, gonadotropin releasing hormone $(\mathrm{GnRH})$ agonist "flare-up" regimens, natural cycle IVF and adding different substances like growth hormone, androgenic agents, aspirin, pyridestigmine, and L-arginine. ${ }^{[9]}$

Also, GnRH antagonist protocol has been advised by several authors as it takes advantages of the initial release of endogenous gonadotropins, giving the chance for recruiting more follicles. ${ }^{[10]}$

Aromatase is a microsomal enzyme (the product of the CYP19 gene) which catalyzes the conversion of androstenedione and testosterone to estrone and estradiol, respectively. ${ }^{[11]}$ Aromatase enzyme is present in many sites not only the ovaries, but also brain, adipose tissue, muscle, liver, breast tissue, and malignant breast tumors. ${ }^{[12]}$

Administration of aromatase inhibitor (AI) in the early days of the menstrual cycle, blocks estrogen production from all sources and release the hypothalamic/pituitary axis from estrogenic negative feedback, without depletion of estrogen receptors (ERs) as occurs with clomiphene citrate (CC). The increase in gonadotropin secretion will stimulate growth of ovarian follicles. ${ }^{[13]}$

Aromatase inhibitors (e.g. letrozole) increases sensitivity of the follicles to follicle stimulating hormone (FSH). This results from temporary accumulation of intraovarian androgens as a result of aromatization which leads to augmentation of FSH receptor expression and follicular growth. ${ }^{[14]}$

Another part of the peripheral action of letrozole is its effect on estrogen receptors (ERs) in the endometrium. Suppression of estrogen levels in the circulation and peripheral target organs results in up-regulation of ERs in the endometrium, which will lead to rapid endometrial growth once estrogen secretion is returned back to normal after letrozole withdrawal. ${ }^{[15]}$

\section{AIM OF THE WORK}

The aim of this work was to study whether adding letrozole to gonadotropin-releasing hormone (GnRH) antagonist protocol would improve the results of intracytoplasmic sperm injection in poor responder patients or not.

\section{PATIENTS AND METHODS}

This randomized controlled study included seventy patients (poor responders) who were recruited and followed up in El-shatby maternity University Hospital and private fertility clinics from December 2017 to November 2018. The diagnosis of poor responders was based on the
Bologna criteria (2011) of the European Society of Human Reproduction and Embryology consensus (ESHRE).

These seventy patients were randomly divided into two groups, group I and group II. Randomization was computer generated by a third party not otherwise involved in the trial; group I included thirty five patients letrozole/ Antagonist group and group II included thirty five patients Antagonist (control) group.

Inclusion Criteria: women 25-40 years old, women with normal ovulatory cycles of 21 to 35 days in length, body mass index (BMI) between 20 and $30 \mathrm{~kg} / \mathrm{m} 2, \mathrm{AMH}$ below $1.1 \mathrm{ng} / \mathrm{dl}$ and all patients were indicated for ICSI

Exclusion Criteria: BMI more than 30, abnormal uterine cavity, hydrosalpinx and endometriosis.

Consent was obtained from all patients to participate in the current work. All cases were subjected to history taking, clinical examination, pelvic ultrasonographic scanning to assess uterine size, shape, antral follicle count (AFC) and $\mathrm{AMH}$ were requested by quantitative enzyme linked immunoassay kit.

Treatment Protocol : In group I: letrozole (Femara, Novartis Pharma AG, Basel, Switzerland) $5 \mathrm{mg}$ per day was administered from the $2^{\text {nd }}$ day to $6^{\text {th }}$ day of menstruation. From the $3^{\text {rd }}$ day of menstruation, 300 IU of human menopausal gonadotropins (HMG) was initiated. In group II: 300 IU of HMG was commenced from the $3^{\text {rd }}$ day of menstruation. GnRH antagonist cetrorelix (Cetrotide, Merck Serono) subcutaneous at a dose $0.25 \mathrm{mg}$ /day was started at day 6 of stimulation and continued daily up to the day of human chorionic gonadotropin injection in both groups.

Response was monitored by serial vaginal ultrasound examinations and evaluation of serum estradiol E2 and (HMG) dose was changed according to response. Human chorionic gonadotropin (10,000 IU) of human chorionic gonadotropin (hCG) (Choriomon $\AA$, IBSA) intra muscular was administered to induce follicular maturation when one or more follicles reached a mean diameter of (17-18 mm) in both groups. Trans-vaginal ultrasound guided oocyte retrieval was performed 34-35 hours after human chorionic gonadotropin injection. Luteal phase support was provided daily from the day after oocyte retrieval, in both groups.

Resulted embryos were graded using the Veeck's score $^{[16]}$ only class I and II embryos were transferred, with a maximum of 4 embryos per transfer. When more than 4 embryos were available for transfer, only 4 of class I or II were transferred and the rest of the embryos were frozen.

Pregnancy was diagnosed by serum beta-human chorionic gonadotropin (B-hcg) 15 days after embryo 
transfer. All patients underwent a trans-vaginal ultrasound scan at 6-7 weeks of pregnancy to differentiate between biochemical and clinical (presence of an intrauterine gestational sac with fetal heart beat) pregnancies and exclusion of ectopic pregnancy.

Outcome measures : 1ry outcome measures; total dose of HMG administered and number of days of stimulation.2ry outcome measures; cancellation rate (cancelled cycles), number of retrieved oocytes, total number of embryos, implantation rate: ratio between number of gestational sacs and the number of transferred embryos.Biochemical pregnancy: serum Beta Human Chorionic Gonadotropin (B-hCG) assay was performed on day 14 after embryo transfer. Clinical pregnancy rate: confirmation of one or more gestational sacs with heart activity by ultrasound 6-7 weeks of gestation.

\section{STATISTICAL ANALYSIS}

Data were fed to the computer and analyzed using IBM SPSS software package version 20.0. (Armonk, NY: IBM Corp) Qualitative data were described using number and percent. Quantitative data were described using range (minimum and maximum), mean, standard deviation and median. Significance of the obtained results was judged at the $5 \%$ level.

\section{RESULTS}

In group I (the letrozole/antagonist group), out of thirty five poor responder patients, only thirty women $(85.7 \%)$ completed the study and five cases discontinued due to cancellation of the stimulation cycle. While in group II (control group) twenty nine women $(82.8 \%)$ only completed the study and six cases cancelled the cycle. The cancellation was due to poor ovarian response to stimulation in both groups.

The demographic data : the age of women in group I ranged from 27 to 40 years with a mean of age (36.0) $\mathrm{SD} \pm 4.33$ years while the age in group II ranged between 25 to 40 years with a mean of age (35.17) $\mathrm{SD} \pm 5.45$ years, with no statistical significant difference. BMI (Body mass index=weight in kilogram/height in meter $\left.^{2}\right)$ in group I ranged between $\left(22.5-29.5 \mathrm{~kg} / \mathrm{m}^{2}\right)$, while in group II it ranged between (22.5-29.3), with no significant statistical difference between both groups. The parity in group I, 25 patients were nulliparous, while ten patients didn't conceive before. In group II the same results were found, with no significant statistical difference. Duration of infertility ranged between (4-15 years) in both groups with no significant statistical difference $(p=0.705)$. Duration of infertility ranged between (4-15 years) in both groups with no significant statistical difference $(p=0.705)$ as shown in (Table 1).
The cancellation rate: In group I five cases canceled the stimulation cycle and 30 women continued the study with cancellation rate $(14.3 \%)$, while in group II six cases stopped the cycle due to poor ovarian response, 29 cases continued the study with cancellation rate $(17.1 \%)$, with no significant statistical difference between the two groups ( ${ }^{\mathrm{FE}} \mathrm{p}=0.743$ ) (Table 2).

Treatment protocol: In group I, thirty poor responder women who continued the study received total HMG dose ranged between $(2400.0$ - 3900.0 I.U) with a mean $(2957.1 \pm 386.0)$. While in group II twenty nine women continued the study and received total dose ranged between (3900.0-5700.0 I.U) with a mean of $(4607.1 \pm 513.9)$, there was significant statistical difference between the two groups $\left(p=<0.001^{*}\right)$. As regards the duration of stimulation, it ranged between (8.0-13.0 days) per patient with mean $=(9.91 \pm 1.27)$ in group I. While in group II, it ranged between (9.0-12.0 days) per patient with mean of duration $(10.52 \pm 0.95)$ with no significant statistical difference between the two groups $(p=0.159)$ (Table 3 ).

ICSI results: In group I, total number of retrieved oocytes ranged between (3.0-11.0) oocytes, with a mean of $(5.97 \pm 2.13)$, while in group II, it ranged between (2.0-9.0 oocytes), with a mean of (6.0 $0 \pm 1.75)$. Regarding MII oocytes per patient in group I the mean was $(5.03 \pm 1.92)$, while in group II it was $(5.45 \pm 1.62)$. According to MI oocytes per patient the mean was $(0.43 \pm 0.77)$ in group I, while it was $(0.21 \pm 0.49)$ in the other group. Number of GV per patient in group I ranged between (0-3 GV), with a mean ( $0.50 \pm 0.94)$, while in group II the mean was $(0.34 \pm 0.67)$. There was no significant statistical difference between both groups as regard total no of retrieved oocytes; MII, M1 and GVs.as shown in (Table 4).

Fertilization rate per patient ranged between (16.67-100\%) in group I, while it ranged between $(28.5-87.5 \%)$ in group II. Total number of embryos per patient, in group I the mean of embryos was $(2.73 \pm 0.83)$, while in group II, it was $(2.86 \pm 0.92)$. Regarding vitrification of embryos per patient, the mean of vitrification in group I $(0.67 \pm 1.15)$, while in group II the mean was $(0.55 \pm 1.12)$ embryo, with no significant statistical difference between the two groups as shown in (Table 5).

Pregnancy rates: Biochemical pregnancy rate in group I was $(20 \%)$, while in group II, it was $(17.2 \%)$. There was no significant statistical difference between the two groups $(p=0.786)$, The clinical pregnancy rate in group I was $(6.7 \%)$, while in group II , it was $(6.9 \%)$. As with biochemical pregnancy, the clinical pregnancy rate was not statistically significant $(p=0.786)$ (Table 6). 
Out of thirty cases in group I, six women had biochemical pregnancy, two of them continued to clinical pregnancy with implantation rate ranged between $(0.0-75.0 \%)$, while in group II, five women had biochemical pregnancy, two of them continued to clinical pregnancy with implantation rate ranged between (0.0-100.0\%), there was no significant statistical difference between the two groups $(p=0.958)$ (Table 7).

Table 1: Comparison between group I and group II according to demographic data

\begin{tabular}{|c|c|c|c|c|c|}
\hline & Data & Group I $(\mathrm{n}=35)$ & Group II $(\mathrm{n}=35)$ & test & $P$ \\
\hline \multirow{3}{*}{ Age (years) } & Min.- Max. & $27.0-40.0$ & $25.0-40.0$ & \multirow{3}{*}{$\mathrm{t}=0.705$} & \multirow{3}{*}{0.483} \\
\hline & Mean \pm SD & $36.0 \pm 4.33$ & $35.17 \pm 5.45$ & & \\
\hline & Median & 37.0 & 36.0 & & \\
\hline \multirow{3}{*}{$\begin{array}{c}\text { BMI } \\
\left(\mathrm{kg} / \mathrm{m}^{2}\right)\end{array}$} & Min. - Max. & $22.50-29.50$ & $22.80-29.30$ & \multirow{3}{*}{$\mathrm{t}=0.358$} & \multirow{3}{*}{0.721} \\
\hline & Mean \pm SD & $26.54 \pm 2.14$ & $26.37 \pm 1.86$ & & \\
\hline & Median & 27.0 & 26.70 & & \\
\hline \multirow{3}{*}{ Parity } & Nulliparous & $25(71.4 \%)$ & $25(71.4 \%)$ & \multirow{3}{*}{$\chi^{2=} 0.00$} & \multirow{3}{*}{1.000} \\
\hline & & & & & \\
\hline & Previous pregnancies & $10(28.6 \%)$ & $10(28.6 \%)$ & & \\
\hline \multirow{3}{*}{$\begin{array}{c}\text { Duration of } \\
\text { infertility }\end{array}$} & Min.- Max. & $4.0-15.0$ & $4.0-15.0$ & \multirow{3}{*}{$\mathrm{U}=580.50$} & \multirow{3}{*}{0.705} \\
\hline & Mean $\pm \mathrm{SD}$ & $8.37 \pm 3.24$ & $8.60 \pm 3.19$ & & \\
\hline & Median & 8.0 & 8.0 & & \\
\hline
\end{tabular}

t: Student t-test

$\chi^{2}$ : Chi square test

$\mathrm{U}$ : Mann Whitney test

$\mathrm{p}$ : $p$ value for comparing between the two groups

Table 2: Comparison between group I and group II according to cancellation rate

\begin{tabular}{|c|c|c|c|c|c|c|}
\hline & \multicolumn{2}{|c|}{$\mathrm{I}(\mathrm{n}=35)$} & \multicolumn{2}{|c|}{ II $(n=35)$} & \multirow{2}{*}{$\chi^{2}$} & \multirow{2}{*}{${ }^{\mathrm{FE}} \mathrm{p}$} \\
\hline & No. & $\%$ & No. & $\%$ & & \\
\hline \multirow[t]{2}{*}{ No Cancellation } & 30 & 85.7 & 29 & 82.9 & & \\
\hline & & & & & 0.108 & 0.743 \\
\hline Cancellation & 5 & 14.3 & 6 & 17.1 & & \\
\hline
\end{tabular}

$\chi^{2}$ : Chi square test

FE: Fisher Exact

$\mathrm{p}$ : $p$ value for comparing between the two groups 
Table 3: Comparison between group I and group II according to total HMG dose (I.U) and duration of simulation

\begin{tabular}{|c|c|c|c|c|c|}
\hline & & $\mathrm{I}(\mathrm{n}=30)$ & II $(\mathrm{n}=29)$ & $\mathrm{T}$ & $P$ \\
\hline \multirow{3}{*}{ Total HMG dose (I.U)/patient } & Min. - Max. & $2400.0-3900.0$ & $3900.0-5700.0$ & \multirow{3}{*}{$15.187^{*}$} & \multirow{3}{*}{$<0.001^{*}$} \\
\hline & Mean \pm SD & $2957.1 \pm 386.0$ & $4607.1 \pm 513.9$ & & \\
\hline & Median & 3000.0 & 4800.0 & & \\
\hline \multirow{3}{*}{ Days of simulation } & Min. - Max. & $8.0-13.0$ & $9.0-12.0$ & \multirow{3}{*}{1.426} & \multirow{3}{*}{0.159} \\
\hline & Mean \pm SD & $10.10 \pm 1.27$ & $10.52 \pm 0.95$ & & \\
\hline & Median & 10.0 & 11.0 & & \\
\hline
\end{tabular}

t: Student t-test

$\mathrm{p}$ : $\mathrm{p}$ value for comparing between the two groups

*: Statistically significant at $p \leq 0.05$

Table 4: Comparison between group I and group II according to total number of oocytes MII, M1 and GV

\begin{tabular}{|c|c|c|c|c|}
\hline & $\mathrm{I}(\mathrm{n}=30)$ & II $(n=29)$ & $\mathrm{U}$ & $P$ \\
\hline \multicolumn{5}{|c|}{ Total number of oocytes } \\
\hline Min. - Max. & $3.0-11.0$ & $2.0-9.0$ & 415.0 & 0.759 \\
\hline Mean \pm SD & $5.97 \pm 2.13$ & $6.0 \pm 1.75$ & & \\
\hline Median & 6.0 & 6.0 & & \\
\hline \multicolumn{5}{|c|}{ Number of MII oocytes } \\
\hline Min. - Max. & $1.0-9.0$ & $2.0-8.0$ & 372.0 & 0.332 \\
\hline Mean $\pm \mathrm{SD}$ & $5.03 \pm 1.92$ & $5.45 \pm 1.62$ & & \\
\hline Median & 5.0 & 5.0 & & \\
\hline \multicolumn{5}{|c|}{ Number of MI oocytes } \\
\hline Min. - Max. & $0.0-2.0$ & $0.0-2.0$ & 385.5 & 0.300 \\
\hline Mean \pm SD & $0.43 \pm 0.77$ & $0.21 \pm 0.49$ & & \\
\hline Median & 0.0 & 0.0 & & \\
\hline \multicolumn{5}{|l|}{ GV } \\
\hline Min. - Max. & $0.0-3.0$ & $0.0-2.0$ & 415.5 & 0.699 \\
\hline Mean \pm SD & $0.50 \pm 0.94$ & $0.34 \pm 0.67$ & & \\
\hline Median & 0.0 & 0.0 & & \\
\hline
\end{tabular}

$\mathrm{U}$ : Mann Whitney test

$\mathrm{p}: p$ value for comparing between the two groups 
Table 5: Comparison between group I and group II according to fertilization rate, total no. of embryos grade1 or2, no of embryos transferred at day 3 and vitrification

$\mathrm{I}(\mathrm{n}=30) \quad \mathrm{II}(\mathrm{n}=29) \quad \mathrm{U} \quad P$

Fertilization rate

Min. - Max.

$16.67-100.0$

$28.57-87.50$

402.0

0.615

Mean \pm SD

$59.03 \pm 23.0$

$56.66 \pm 17.50$

66.67

60.0

Total no. of embryos grade1

Min. - Max.

Mean \pm SD

$1.0-7.0$

$1.0-7.0$

423.0

0.852

Median

3.0

$3.41 \pm 1.57$

3.0

No of embryos transferred at day 3

Min. - Max.

$1.0-4.0$

398.0

Mean \pm SD

$2.73 \pm 0.83$

$2.86 \pm 0.92$

Median

3.0

3.0

Vitrification rate

Min. - Max.

$0.0-3.0$

$0.0-3.0$

413.0

0.654

Mean \pm SD

$0.67 \pm 1.15$

$0.55 \pm 1.12$

Median

0.0

0.0

U: Mann Whitney test

$\mathrm{p}: p$ value for comparing between the two groups

Table 6: Comparison between the group I and group II according to different pregnancy rates

\begin{tabular}{|c|c|c|c|c|c|c|c|}
\hline \multirow{2}{*}{\multicolumn{2}{|c|}{ Pregnancy rate }} & \multicolumn{2}{|c|}{$\mathrm{I}(\mathrm{n}=30)$} & \multicolumn{2}{|c|}{ II (n=29) } & \multirow{2}{*}{ test } & \multirow{2}{*}{$\mathrm{P} /{ }^{\mathrm{FE}} \mathrm{p}$} \\
\hline & & No. & $\%$ & No. & $\%$ & & \\
\hline \multirow[t]{2}{*}{ biochemica } & No & 24 & 80.0 & 24 & 82.8 & $\chi^{2}=0.074$ & 0.786 \\
\hline & Yes & 6 & 20.0 & 5 & 17.2 & & \\
\hline \multirow[t]{2}{*}{ clinical } & No & 28 & 93.3 & 27 & 93.1 & $\chi^{2}=0.001$ & 1.000 \\
\hline & Yes & 2 & 6.7 & 2 & 6.9 & & \\
\hline
\end{tabular}

$\chi^{2}$ : Chi square test

FE: Fisher Exact

$\mathrm{p}$ : $p$ value for comparing between the two groups

Table 7: Comparison between the group I and group II according to gestational sacs at 6 week and implantation rate

\begin{tabular}{lccc}
\hline & $\mathrm{I}(\mathrm{n}=2)$ & $\mathrm{II}(\mathrm{n}=2)$ & $\mathrm{U}$ \\
\hline & & & \\
Gestational sacs & & & \\
Min. - Max. & $0.0-3.0$ & $0.0-2.0$ & 435.0 \\
Mean \pm SD. & $0.17 \pm 0.65$ & $0.14 \pm 0.52$ & 0.0 \\
Median & 0.0 & & \\
& & & 4.000 \\
Implantation rate & & & 433.50 \\
Min. - Max. & $0.0-75.0$ & $0.0-100.0$ \\
Mean \pm SD. & $4.72 \pm 18.0$ & $5.75 \pm 21.95$ \\
Median & 0.0 & 0.0 & 0.958 \\
\hline
\end{tabular}

U: Mann Whitney test

$\mathrm{p}: p$ value for comparing between the two groups

Implantation rate*: (No. of gestational sacs/ No. of embryos transferred)*100 


\section{DISCUSSION}

Poor responders represent an important and less fortunate group of patients attending any ICSI clinic, as they have a higher cancellation rate and lower implantation and clinical pregnancy rates. Up till now, there is no consensus on the best method for the prediction and/or management of this group.

Our study aimed to evaluate the potential role of aromatase inhibitors (letrozole) as an adjuvant drug to improve the ICSI cycle outcomes of standard GnRH antagonist stimulation protocol in those patients.

The result of the study showed no significant difference in the number of oocytes retrieved, cycle cancelation rate, clinical pregnancy rate and implantation rate, between both groups, but there was significant difference regarding the total HMG dose which was significantly decreased in letrozole group.

Reduced pregnancy outcome in poor responders may be attributed to the effect of short follicular phase and low FSH receptor expression in granulosa cells ${ }^{[17]}$. Ovarian response to stimulation protocol is enhanced by the letrozolemediated decrease in serum estrogen levels and temporary enhance in intra ovarian androgen concentrations cause prolongation of the follicular phase, increase in affinity of FSH receptors, preantral and antral follicles growth. ${ }^{[17,18]}$ It might be due to letrozole- induced PCO-like condition and an increase in pre antral and antral folliclar numbers. ${ }^{[19]}$

The use of aromatase inhibitors in a GnRH antagonist protocol was suggested by some studies. Yarali and colleagues demonstrated that adjuvant therapy with letrozole could improve the response in poor responder patients $^{[20]}$.

According to the literature, there are different studies with conflicting results, some showed that adding letrozole decreased the doses of HMG and days of stimulation (Lee, Kyung-Hee, et al. $)^{[21]}$, others found significant increase in oocytes numbers and no significant increase in fertilization rate with letrozole group(Chung et al. $)^{[22]}$,(Ozmen et al.) found that adding letrozole had benefits in improving the success of ovarian stimulation cycles by decreasing cycle cancellation rate. ${ }^{[23]}$ (Mahbod Ebrahimi et al) found no significant difference regarding implantation rate after giving aromatase inhibitors to their patients. ${ }^{[24]}$

Jeve et al., ${ }^{[25]}$ a systemic review aimed to identify the most effective treatment protocol for poor response patients. They searched MEDLINE, EMBASE, and The Cochrane Library from 1980 to October 2015 and found four trials; $(n=223)$ comparing aromatase inhibitors in poor responders. They said that adding letrozole has no effect on oocyte yield, cancellation rate and pregnancy rates in cases of poor responders doing an ICSI cycle.

\section{CONCLUSION}

Lerrozole is recommended to be added to ICSI stimulation protocols in poor responders. Although it didn't affect the pregnancy rate, letrozole decreases the dose and the cost of ICSI cycles. . This could make assisted reproductive technology more friendly and cheaper to those patients.

\section{CONFLICT OF INTERESTS}

There are no conflicts of interests.

\section{REFERENCES}

1. Sallam HN, Ezzeldin F, Agameya AF, Rahman AF, El-Garem Y. Defining poor responders in assisted reproduction. Int J Fertil Women's Med 2005; 50:115-20.

2. Surrey ES, Schoolcraft WB. Evaluating strategies for improving ovarian response of the poor responder undergoing assisted reproductive techniques. Fertil Steril 2000; 73:667-76.

3. Shastri SM, Barbieri E, Kligman I, Schoyer KD, Davis OK, Rosenwaks Z. Stimulation of the young poor responder: comparison of the luteal estradiol/ gonadotropin-releasing hormone antagonist priming protocol versus oral contraceptive microdose leuprolide. Fertil Steril 2011; 95(2):592-5.

4. Zarek SM, Muasher SJ. Mild/minimal stimulation for in vitro fertilization: an old idea that needs to be revisited. Fertil Steril 2011; 95(8):2449-55.

5. Mutlu MF, Erdem M, Erdem A, Yildiz S, Mutlu I, Arisoy $\mathrm{O}$, et al. Antral follicle count determines poor ovarian response better than anti- fertilization cycles. J Assisted Reprod Genet 2013; 30(5):657-65.

6. Ferraretti AP, La Marca A, Fauser BC, Tarlatzis B, Nargund G, Gianaroli L, et al. ESHRE consensus on the definition of 'poor response'to ovarian stimulation for in vitro fertilization: the Bologna criteria. Hum Reprod 2011; 26(7):1616-24.

7. Broer SL, van Disseldorp J, Broeze KA, Dolleman $\mathrm{M}$, Opmeer $\mathrm{BC}$, Bossuyt $\mathrm{P}$, et al. Added value of ovarian reserve testing on patient characteristics in the prediction of ovarian response and ongoing pregnancy: an individual patient data approach. Hum Reprod Update 2013; 19(1):26-36.

8. Verberg MF, Eijkemans MJ, Heijnen EM, Broekmans FJ, de Klerk C, Fauser BC, et al. Why do couples drop-out from IVF treatment? A prospective cohort study. Hum Reprod 2008; 23(9):2050-55. 
9. Ubaldi FM, Rienzi L, Ferrero S, Baroni E, Sapienza $\mathrm{F}$, Cobellis L, et al. Management of poor responders in IVF, “ (in eng), Reprod Biomed Online 2005; $10(2): 235-46$.

10. Kolibianakis E, Albano C, Zikopoulos K, Kahn JA, Steirteghem AV, Devroey P. GnRH antagonists in poor responders. Acta Obstetr Gynecol Scand 2004; 83(12):1216-7

11. Akhtar M, Njar VC, Wright JN. Mechanistic studies on aromatase and related $\mathrm{C}-\mathrm{C}$ bond cleaving P-450 enzymes. J Steroid Biochem Mol Biol 1993; 44(4):375-87.

12. Vanden Bossche HV, Moereels H, Koymans LM. Aromatase inhibitors-mechanisms for non-steroidal inhibitors. Breast Cancer Res Treat 1994; 30(1):43-55.

13. Sioufi A, Gauducheau N, Pineau V, Marfil F, Jaouen A, Cardot JM, et al. Absolute bioavailability of letrozole in healthy postmenopausal women. Biopharm Drug Dispos 1997; 18(9):779-89.

14. Vendola K, Zhou J, Wang J, Famuyiwa OA, Bievre M, Bondy CA. Androgens Promote Oocyte Insulin-Like Growth Factor I Expression and Initiation of Follicle Development in the Primate Ovary. Biol Reprod 1999; 61(2):353-7.

15. Al-Fozan H, Al-Khadouri M, Tan SL, Tulandi T. A randomized trial of letrozole versus clomiphene citrate in women undergoing superovulation. Fertil Steril 2004; 82(6):1561-3.

16. Veeck LL. Atlas of the human oocyte and Early Conceptus. Baltimore: Williams and Wilkins; 1991. p.427-44

17. Garcia-Velasco JA, Moreno L, Pacheco A, Guillen A, Duque L, Requena A, et al. The aromatase inhibitor letrozole increases the concentration of intraovarian androgens and improves in vitro fertilization outcome in low responder patients: a pilot study. Fertil Steril $2005 ; 84: 82-7$
18. Goswami SK, Das T, Chattopadhyay R, Sawhney V, Kumar J, Chaudhury $\mathrm{K}$, et al. A randomized singleblind controlled trial of letrozole as a low-cost IVF protocol in women with poor ovarian response: a preliminary report. Hum Reprod 2004; 19(9):2031-5.

19. Demeestere I, Gervy C, Centner J, Devreker F, Englert Y, Delbaere A. Effect of insulin-like growth factor-I during preantral follicular culture on steroidogenesis, in vitro oocyte maturation, and embryo development in mice. Biol Reprod 2004; 70(6):1664-9

20. Yarali H, Esinler İ, Polat M, Bozdag G, Tiras B. Antagonist/letrozole protocol in poor ovarian responders for intracytoplasmic sperm injection: a comparative study with the microdose flare-up protocol. Fertil Steril 2009; 92(1):231-5.

21. Lee KH, Kim CH, Suk HJ, Lee YJ, Kwon SK, Kim $\mathrm{SH}$, et al. The Effect of Aromatase Inhibitor Letrozole Incorporated in Gonadotrophin-Releasing Hormone Antagonist Multiple Dose Protocol in Poor Responders Undergoing in Vitro Fertilization. Obstetr Gynecol Sci 2014; 57(3):216-22.

22. Chung MK, Ryu MJ, Kim HS, Kim MH, Jeong HJ, Lee HS, et al. Treatment of aromatase inhibitors in severe poor responder patients: matched comparison with non-treated previous cycles in same patients. Fertil Steril 2014; 102(3):e66-1.

23. Ozmen B, Sonmezer M, Atabekoglu CS, Olmus H Use of aromatase inhibitors in poor-responder patients receiving GnRH antagonist protocol. Reprod BioMed Online 2009; 19(4):478-85.

24. Ebrahimi M, Akbari-Asbagh F, Ghalandar-Attar M. Letrozole + GnRH antagonist stimulation protocol in poor ovarian responders undergoing intracytoplasmic sperm injection cycles: An RCT. Int J Reprod Biomed 2017; 15(2):101-8.

25. Jeve YB, Bhandari HM. Effective treatment protocol for poor ovarian response: A systematic review and meta-analysis. J Hum Reprod Sci 2016; 9(2):70-81. 